\title{
A Comparison of Three Total Variation Based Texture Extraction Models *
}

\author{
Wotao Yin ${ }^{\mathrm{a}}$, Donald Goldfarb ${ }^{\mathrm{b}}$, Stanley Osher ${ }^{\mathrm{c}}$ \\ ${ }^{a}$ Rice University, Department of Computational and Applied Mathematics, 6100 \\ Main St, MS-134, Houston, TX 77005, USA \\ ${ }^{\mathrm{b}}$ Columbia University, Department of Industrial Engineering and Operations \\ Research, 500 West 120th St, Mudd 313, New York, NY 10027, USA \\ ${ }^{\mathrm{c}}$ UCLA Mathematics Department, Box 951555, Los Angeles, CA 90095, USA
}

\begin{abstract}
This paper qualitatively compares three recently proposed models for signal/image texture extraction based on total variation minimization: the Meyer [27], Vese-Osher (VO) [35], and TV- $L^{1}[12,38,2-4,29-31]$ models. We formulate discrete versions of these models as second-order cone programs (SOCPs) which can be solved efficiently by interior-point methods. Our experiments with these models on 1D oscillating signals and 2D images reveal their differences: the Meyer model tends to extract oscillation patterns in the input, the TV- $L^{1}$ model performs a strict multiscale decomposition, and the Vese-Osher model has properties falling in between the other two models.
\end{abstract}

Key words: image decomposition, texture extraction, feature selection, total variation, variational imaging, second-order cone programming, interior-point method

\section{Introduction}

Let $f$ be an observed image that contains texture and/or noise. Texture is characterized as repeated and meaningful structure of small patterns. Noise is

\footnotetext{
‡ Research supported by NSF Grants DMS-01-04282, DNS-03-12222 and ACI-0321917, ONR Grants N00014-03-1-0514 and N00014-03-0071, and DOE Grant GEFG01-92ER-25126.

Email addresses: wotao.yin@rice.edu (Wotao Yin), goldfarb@columbia.edu (Donald Goldfarb), sjo@math.ucla.edu (Stanley Osher).
} 
characterized as uncorrelated random patterns. The rest of an image, which is called cartoon, contains object hues and sharp edges (boundaries). Thus an image $f$ can be decomposed as $f=u+v$, where $u$ represents image cartoon and $v$ is texture and/or noise. A general way to obtain this decomposition using the variational approach is to solve the problem $\min \left\{T V(u) \mid\|u-f\|_{B} \leq \sigma\right\}$, where $T V(u)$ denotes the total variation of $u$ and $\|\cdot\|_{B}$ is a norm (or seminorm). The total variation of $u$, which is defined below in Subsection 1.1, is minimized to regularize $u$ while keeping edges like object boundaries of $f$ in $u$ (i.e., while allowing discontinuities in $u$ ). The fidelity term $\|u-f\|_{B} \leq \sigma$ forces $u$ to be close to $f$.

\subsection{The spaces $B V$ and $G$}

The Banach space $B V$ of functions of bounded variation is important in image processing because such functions are allowed to have discontinuities and hence keep edges. This can be seen from its definition as follows.

Let $u \in L^{1}$, and define [39]

$$
T V(u):=\sup \left\{\begin{array}{ll}
u \operatorname{div}(\vec{g}) d x: \begin{array}{l}
\vec{g} \in C_{0}^{1}\left(\mathbb{R}^{n} ; \mathbb{R}^{n}\right), \\
|\vec{g}(x)| \leq 1 \text { for all } x \in \mathbb{R}^{n}
\end{array}
\end{array}\right\}
$$

as the total variation of $u$, where $|\cdot|$ denotes the Euclidean norm. Also, $u \in B V$ if $\|u\|_{B V}:=\|u\|_{L^{1}}+T V(u)<\infty$. In the above definition, $\vec{g} \in C_{0}^{1}\left(\mathbb{R}^{n} ; \mathbb{R}^{n}\right)$, the set of continuously differentiable vector-valued functions, serves as a test set for $u$. If $u$ is in the Sobolev spaces $W^{1,1}$ or $H^{1}$, it follows from integration by parts that $T V(u)$ is equal to $\int|\nabla u|$, where $\nabla u$ is the weak derivative of $u$. However, the use of test functions to define $T V(u)$ allows $u$ to have discontinuities. Therefore, $B V$ is larger than $W^{1,1}$ and $H^{1}$. Equipped with the $\|\cdot\|_{B V^{-n o r m},} B V$ is a Banach space.

$B V(\Omega)$ with $\Omega$ being a bounded open domain is defined analogously to $B V$ with $L^{1}$ and $C_{0}^{1}\left(\mathbb{R}^{n} ; \mathbb{R}^{n}\right)$ replaced by $L^{1}(\Omega)$ and $C_{0}^{1}\left(\Omega ; \mathbb{R}^{n}\right)$, respectively.

Next, we define the space $G$ [27]. Let $G$ denote the Banach space consisting of all generalized functions $v(x)$ defined on $\mathbb{R}^{n}$, which can be written as

$$
v=\operatorname{div}(\vec{g}), \quad \vec{g}=\left[g_{i}\right]_{i=1, \ldots, n} \in L^{\infty}\left(\mathbb{R}^{n} ; \mathbb{R}^{n}\right) .
$$

Its norm $\|v\|_{G}$ is defined as the infimum of all $L^{\infty}$ norms of the functions $|\vec{g}(x)|$ over all decompositions (1) of $f$. In short, $\|v\|_{G}=\inf \left\{\|(|\vec{g}(x)|)\|_{L^{\infty}}\right.$ : $v=\operatorname{div}(\vec{g})\}$. $G$ is the dual of the closed subspace $\mathcal{B V}$ of $B V$, where $\mathcal{B V}:=$ $\left\{u \in B V:|\nabla f| \in L^{1}\right\}[27]$. We note that finite difference approximations to 
functions in $B V$ and $\mathcal{B V}$ are the same. For the definition and properties of $G(\Omega)$, see $[5]$.

An immediate result of the above definitions is that

$$
\int u v=\int u \nabla \cdot \vec{g}=-\int \nabla u \cdot \vec{g} \leq T V(u)\|v\|_{G},
$$

holds for any $u \in \mathcal{B} \mathcal{V}$ with compact support and $v \in G$. We say $(u, v)$ is an extremal pair if (2) holds with equality.

In image processing, the space $B V$ and the total variation semi-norm were first used by Rudin, Osher, and Fatemi [33] to remove noise from images. Specifically, their model obtains a cleaner image $u \in B V$ of a noisy image $f$ by letting $u$ be the minimizer of $T V(u)+\lambda\|u-f\|_{L^{2}}^{2}$, in which the regularizing term $T V(u)$ tends to reduce the oscillations in $u$ and the data fidelity term $\|u-f\|_{L^{2}}$ tends to keep $u$ close to $f$.

The ROF model is the precursor to a large number of image processing models having a similar form. Among the recent total variation-based cartoon-texture decomposition models, Meyer [27] and Haddad and Meyer [20] proposed using the $G$-norm defined above, Vese and Osher [35] approximated the $G$-norm by the $\operatorname{div}\left(L^{p}\right)$-norm, Osher, Sole and Vese [32] proposed using the $H^{-1}$-norm, Lieu and Vese [26] proposed using the more general $H^{-s}$-norm, and Le and Vese [24] and Garnett, Le, Meyer and Vese [18] proposed using the homogeneous Besov space $\dot{B}_{p, q}^{s},-2<s<0,1 \leq p, q \leq \infty$, extending Meyer's $\dot{B}_{\infty, \infty}^{-1}$, to model the oscillation component of an image. In addition, Chan and Esedoglu [12] and Yin, Goldfarb and Osher [38] used the $L^{1}$-norm together with total variation, following the earlier work by Alliney [2-4] and Nikolova [29-31].

\subsection{Three cartoon-texture decomposition models}

In this subsection we present three cartoon-texture decomposition models that are based on the minimization of total variation. We suggest that readers interested in the theoretical analysis of these models read the referenced works mentioned below and in the introduction. Although the analysis of the existence and uniqueness of solutions and duality/conjugacy is not within the scope of our discussion, in Section 3 we relate the differences among the image results from these models to the distinguished properties of the three fidelity terms: $\|f-u\|_{L^{1}},\|f-u\|_{G}$, and its approximation by Vese and Osher.

In the rest of the paper, we assume the input image $f$ has compact support contained in a bounded convex open set $\Omega$. In our tests, $\Omega$ is an open square. 


\subsubsection{The TV-L $L^{1}$ model}

In $[2-4,30,31,12,37]$ the square of the $L^{2}$ norm of $f-u$ in the fidelity term in the original ROF model $\left(\min \left\{T V(u)+\lambda\|f-u\|_{L^{2}}^{2}\right\}\right)$ is replaced by the $L^{1}$ norm of $f-u$, which yields the following problem:

$$
\begin{aligned}
& \text { Constraint model: } \min _{u \in B V}\left\{\int_{\Omega}|\nabla u|, \text { s.t. } \int|f-u| \leq \sigma\right\}, \\
& \text { Lagrangian model: } \min _{u \in B V} \int_{\Omega}|\nabla u|+\lambda \int|f-u| .
\end{aligned}
$$

The above constrained minimization problem (3) is equivalent to its Lagrangian relaxed form (4), where $\lambda$ is the Lagrange multiplier of the constraint $\int|f-u|$. The two problems have the same solution if $\lambda$ is chosen equal to the optimal value of the dual variable corresponding to the constraint in the constrained problem. Given $\sigma$ or $\lambda$, we can calculate the other value by solving the corresponding problem. The same result also holds for Meyer's model below.

We chose to solve the Lagrangian relaxed version (4), rather than the constraint version (3), in our numerical experiments because several researchers $[12,37]$ have established the relationship between $\lambda$ and the scale of $f-u^{*}$. For example, for the unit disk signal $\mathbf{1}_{B(0, r)}$ centered at origin and with radius $r$, $f-u^{*}=\mathbf{1}_{B(0, r)}$ for $0<\lambda<2 / r$ while $f-u^{*}$ vanishes for $\lambda>2 / r$. Although this model appears to be simpler than Meyer's model and the Vese-Osher model below, it has recently been shown to have very interesting properties like morphological invariance and texture extraction by scale [12,37]. These properties are important in various applications in biomedical engineering and computer vision such as background correction [36], face recognition [14,15], and brain MR image registration [13]. In Section 3, we demonstrate the ability of the TV- $L^{1}$ model to separate out features of a certain scale in an image.

In addition to the SOCP approach that we use in the paper to solve (4) numerically, the graph-based approaches $[17,11]$ were recently demonstrated very efficient in solving an approximate version of (4).

\subsubsection{Meyer's model}

To extract cartoon $u$ in the space $B V$ and texture and/or noise $v$ as an oscillating function, Meyer [27] proposed the following model:

$$
\begin{aligned}
& \text { Constraint version: } \inf _{u \in B V}\left\{\int|\nabla u| \text {, s.t. }\|f-u\|_{G} \leq \sigma\right\}, \\
& \text { Lagrangian version: } \inf _{u \in B V} \int|\nabla u|+\lambda\|f-u\|_{G} .
\end{aligned}
$$


As we have pointed out in Section 1.1, $G$ is the dual space of $\mathcal{B V}$, a subspace of $B V$. So $G$ is closely connected to $B V$. Meyer gave a few examples, including the one shown at the end of next paragraph, in [27] illustrating the appropriateness of modeling oscillating patterns by functions in $G$.

Unfortunately, it is not possible to write down Euler-Lagrange equations for the Lagrangian form of Meyer's model (6), and hence, use a straightforward partial differential equation method to solve it. Alternatively, several models $[5,6,32,35]$ have been proposed to solve (6) approximately. The Vese-Osher model [35] described in the next subsection approximates $\|(|\vec{g}(x)|)\|_{L^{\infty}}$ by $\|(|\vec{g}(x)|)\|_{L^{p}}$, with $1 \leq p<\infty$. The Osher-Sole-Vese model [32] replaces $\|v\|_{G}$ by the Hilbert functional $\|v\|_{H^{-1}}^{2}$. The more recent $\mathrm{A}^{2} \mathrm{BC}$ model $[5,7,6]$ is inspired by Chambolle's projection algorithm [10] and minimizes $T V(u)+$ $\lambda\|f-u-v\|_{L^{2}}^{2}$ for $(u, v) \in B V \times\left\{v \in G:\|v\|_{G} \leq \mu\right\}$. Similar projection algorithms proposed in [9] and [8] are also used to approximately solve (4) and (6). Recently, Kindermann and Osher [21] showed that (6) is equivalent to a minimax problem and proposed a numerical method to solve this saddle-point problem. Other numerical approaches based on the dual representation of the $G$-norm are introduced in [16] by Chung, Le, Lieu, Tanushev, and Vese, [25] by Lieu, and [23] by Le, Lieu, and Vese. In [34], Starck, Elad, and Donoho use sparse basis pursuit to achieve a similar decomposition. In Section 2, we present SOCP-based optimization models to solve both (5) and (6) exactly (i.e., without any approximation or regularization applied to the non-smooth terms $\int|\nabla u|$ and $\|v\|_{G}$ except for the use of finite differences). In contrast to our choice for the TV- $L^{1}$ model, we chose to solve (5) with specified $\sigma$ 's in our numerical experiments because setting an upper bound on $\|f-u\|_{G}$ is more meaningful than penalizing $\|f-u\|_{G}$. The following example demonstrates that $\|v\|_{G}$ is inversely proportional to the oscillation of $v$ : let $v(t)=\cos (x t)$, which has stronger oscillations for larger $t$; one can show $\|v\|_{G}=1 / t$ because $\cos (x t)=\frac{d\left(\frac{1}{t} \sin (x t)\right)}{d x}$ and $\left\|\frac{1}{t} \sin (x t)\right\|_{L^{\infty}}=1 / t$. Therefore, to separate a signal with oscillations stronger than a specific level from $f$, it is more straightforward to solve the constrained problem (5).

To calculate the $G$-norm of a function $f$ alone, one can choose to solve an SOCP or use the dual method by Kindermann, Osher and Xu [22]. The authors of the latter work exploit (2) to develop a level-set based iterative method.

\subsubsection{The Vese-Osher model}

Motivated by the definition of the $L^{\infty}$ norm of $|\vec{g}(x)|$ as the limit

$$
\|(|\vec{g}|)\|_{L^{\infty}}=\lim _{p \rightarrow \infty}\|(|\vec{g}|)\|_{L^{p}},
$$


Vese and Osher [35] proposed the following approximation to Meyer's model (5):

$$
\inf _{u \in B V, \vec{g} \in C_{0}^{1}\left(\mathbb{R}^{n} ; \mathbb{R}^{n}\right)}\left\{V O_{p}(u, \vec{g}):=\int|\nabla u|+\lambda \int|f-u-\operatorname{div}(\vec{g})|^{2}+\mu\left[\int|\vec{g}|^{p}\right]^{1 / p}\right\},
$$

where $p \geq 1$.

In $\mathbb{R}^{2}$, minimizing $V O_{p}$ with respect to $u, \vec{g}=\left(g_{1}, g_{2}\right)$ yields the associated Euler-Lagrange equations:

$$
\begin{array}{r}
u=f-\partial_{1} g_{1}-\partial_{2} g_{2}+\frac{1}{2 \lambda} \operatorname{div}\left(\frac{\nabla u}{|\nabla u|}\right), \\
\mu\left(\left\|\sqrt{g_{1}^{2}+g_{2}^{2}}\right\|_{L^{p}}\right)^{1-p}\left(\sqrt{g_{1}^{2}+g_{2}^{2}}\right)^{p-2} g_{1}=2 \lambda\left[\partial_{1}(u-f)+\partial_{11}^{2} g_{1}+\partial_{12}^{2} g_{2}\right], \\
\mu\left(\left\|\sqrt{g_{1}^{2}+g_{2}^{2}}\right\|_{L^{p}}\right)^{1-p}\left(\sqrt{g_{1}^{2}+g_{2}^{2}}\right)^{p-2} g_{2}=2 \lambda\left[\partial_{2}(u-f)+\partial_{12}^{2} g_{1}+\partial_{22}^{2} g_{2}\right] .
\end{array}
$$

In [35], the authors solve the above system of partial differential equations for different values of $p$, with $1 \leq p \leq 10$, via a sequential descent approach and claim that they give very similar numerical results.

The VO model (8) can be viewed as a relaxation of Meyer's model (5) since the requirement $f-u=\operatorname{div}(g)$ is relaxed by penalizing its violation and $\sup _{\vec{g}}\left\{\|(|\vec{g}|)\|_{L^{\infty}}:\|(|\vec{g}|)\|_{L^{p}} \leq \sigma\right\}=\infty$. This point is clearly illustrated by the numerical comparisons between these two models presented in Section 3.

\subsection{Second-order cone programming}

The purpose of this paper is to accurately compute and compare the three TV-based models presented above using a uniform approach. To do this we formulate and solve all of the above three models as second-order cone programs (SOCPs). These formulations do not require the use of regularization to handle the non-smoothness of these models. In this subsection, we give a short introduction to SOCP and the use of interior-point methods to solve SOCPs.

In an SOCP the vector of variables $\mathbf{x} \in \mathbb{R}^{n}$ is composed of subvectors $\mathbf{x}_{\mathbf{i}} \in \mathbb{R}^{n_{i}}$ - i.e., $\mathbf{x} \equiv\left(\mathbf{x}_{\mathbf{1}} ; \mathbf{x}_{\mathbf{2}} ; \ldots ; \mathbf{x}_{\mathbf{r}}\right)$ - where $n=n_{1}+n_{2}+\ldots+n_{r}$ and each subvector 
$\mathbf{x}_{\mathbf{i}}$ must lie either in an elementary second-order cone of dimension $n_{i}$

$$
\mathcal{K}^{n_{i}} \equiv\left\{\mathbf{x}_{\mathbf{i}}=\left(x_{i}^{0} ; \overline{\mathbf{x}}_{\mathbf{i}}\right) \in \mathbb{R} \times \mathbb{R}^{n_{i}-1}|| \overline{\mathbf{x}}_{\mathbf{i}} \mid \leq x_{i}^{0}\right\}
$$

or an $n_{i}$-dimensional rotated second-order cone

$$
\mathcal{Q}^{n_{i}} \equiv\left\{\mathbf{x}_{\mathbf{i}} \in \mathbb{R}^{n_{i}} \mid \mathbf{x}_{\mathbf{i}}=\overline{\mathbf{x}}, 2 \bar{x}_{1} \bar{x}_{2} \geq \sum_{i=3}^{n_{i}} \bar{x}_{i}^{2}, \bar{x}_{1}, \bar{x}_{2} \geq 0\right\}
$$

Note $\mathcal{Q}^{n_{i}}$ is an elementary second-order cone under a linear transformation; i.e.,

$\left(\frac{1}{\sqrt{2}}\left(x_{1}+x_{2}\right) ; \frac{1}{\sqrt{2}}\left(x_{1}-x_{2}\right) ; x_{3} ; \ldots ; x_{n_{i}}\right) \in \mathcal{K}^{n_{i}} \Longleftrightarrow\left(x_{1} ; x_{2} ; x_{3} ; \ldots ; x_{t}\right) \in \mathcal{Q}^{n_{i}}$

With these definitions an SOCP can be written in the following form [1]:

$$
\begin{array}{ll}
\min & \mathbf{c}_{\mathbf{1}}^{\top} \mathbf{x}_{\mathbf{1}}+\cdots+\mathbf{c}_{\mathbf{r}}^{\top} \mathbf{x}_{\mathbf{r}} \\
\text { s.t. } & A_{1} \mathbf{x}_{\mathbf{1}}+\cdots+A_{r} \mathbf{x}_{\mathbf{r}}=\mathbf{b} \\
& \mathbf{x}_{\mathbf{i}} \in \mathcal{K}^{n_{i}} \text { or } \mathcal{Q}^{n_{i}}, \quad \text { for } i=1, \ldots, r
\end{array}
$$

where $\mathbf{c}_{\mathbf{i}} \in \mathbb{R}^{n_{i}}$ and $A_{i} \in \mathbb{R}^{m \times n_{i}}$, for $i=1, \ldots, r$ and $\mathbf{b} \in \mathbb{R}^{m}$.

Since a one-dimensional second-order cone corresponds to a semi-infinite ray, SOCPs can accommodate nonnegative variables. In fact if all cones are onedimensional, then the above SOCP is just a standard form linear program. As is the case for linear programs, SOCPs can be solved in polynomial time by interior point methods. This is the approach that we take to solve the TV-based cartoon-texture decomposition models in this paper.

\subsection{Interior-point methods for SOCPs}

Over the past two decades there has been extensive research on and development of the interior-point methods for solving linear programs. In the last few years this research and development has been extended to SOCPs. Consequently, these problems can now be solved efficiently both in practice and in theory (in polynomial time). Moreover, interior-point SOCP methods often yield highly accurate solutions. The optimality conditions for the SOCP (13) 
are

$$
\begin{aligned}
A_{1} \mathbf{x}_{\mathbf{1}}+\cdots+A_{r} \mathbf{x}_{\mathbf{r}} & =\mathbf{b}, \\
A_{i}^{\top} \mathbf{y}+\mathbf{s}_{\mathbf{i}} & =\mathbf{c}_{\mathbf{i}}, \text { for } i=1, \ldots, r, \\
\mathbf{x}_{\mathbf{i}}^{\top} \mathbf{s}_{\mathbf{i}} & =0, \text { for } i=1, \ldots, r, \\
x_{i}^{0} \overline{\mathbf{s}_{\mathbf{i}}}+s_{i}^{0} \overline{\mathbf{x}}_{\mathbf{i}} & =\mathbf{0}, \text { for } i=1, \ldots, r .
\end{aligned}
$$

Interior-point methods for SOCPs approximately solve a sequence of perturbed optimality conditions (the "0" on the right-hand side of the third block in (14), which is equal to the duality gap, is replaced by a positive scalar $\mu$ ) by taking single damped Newton steps while making sure that the new iterates remain interior (i.e., $\mathbf{x}_{\mathbf{i}}+\Delta \mathbf{x}_{\mathbf{i}}$ and $\mathbf{s}_{\mathbf{i}}+\Delta \mathbf{s}_{\mathbf{i}}$ are strictly inside their respective cones). The iteration stops once a new iterate satisfies certain prescribed stopping conditions such as the duality gap $\mu$ falling below a tolerance. The typical number of iterations is between 15 and 50 and is usually fairly independent of the problem size. Moreover, in [19] it is shown that each interior-point iteration takes $O\left(n^{3}\right)$ time and $O\left(n^{2} \log n\right)$ bytes for solving an SOCP formulation of the Rudin-Osher-Fatemi model [33].

\section{Formulating the models as SOCPs}

\subsection{Preliminaries}

In practice grey-scale images are represented as 2-dimensional matrices, whose elements give the grey-scale values of corresponding pixels. In this paper we restrict our discussion to square domains in $\mathbb{R}^{2}$ and hence $n \times n$ real matrices (denoted by $M^{n \times n}$ ) for the sake of simplicity.

Let $f \in M^{n \times n}$ be an observed image and let $u$ denote the cartoon and $v$ denote the texture and/or noise in $f$ such that $(f, u, v)$ satisfies

$$
f_{i, j}=u_{i, j}+v_{i, j}, \quad \text { for } i, j=1, \ldots, n \text {. }
$$

$f_{i, j}, u_{i, j}$, and $v_{i, j}$ are, respectively, the grey-scale values of the observed image, the cartoon, and the texture/noise at pixel $(i, j)$.

All of the three models considered in this paper minimize the total variation of $u, T V(u)$, to regularize $u$. Here we present a discretization scheme and convert $\min T V(u)$ into an SOCP. First, we use forward finite differences to define the total variation of $u$ as follows:

$$
\mathrm{TV}(u) \stackrel{\text { def }}{=} \sum_{1 \leq i, j \leq n}\left|\partial^{+} u_{i, j}\right|
$$


where $|\cdot|$ denotes the Euclidean norm, i.e., $\left|\partial^{+} u_{i, j}\right|=\left(\left(\left(\partial_{x}^{+} u\right)_{i, j}\right)^{2}+\left(\left(\partial_{y}^{+} u\right)_{i, j}\right)^{2}\right)^{1 / 2}$, and $\partial^{+}$denotes the discrete differential operator defined by

$$
\partial^{+} u_{i, j} \stackrel{\text { def }}{=}\left(\left(\partial_{x}^{+} u\right)_{i, j},\left(\partial_{y}^{+} u\right)_{i, j}\right)
$$

where

$$
\begin{aligned}
& \left(\partial_{x}^{+} u\right)_{i, j} \stackrel{\text { def }}{=} u_{i+1, j}-u_{i, j}, \quad \text { for } i=1, \ldots, n-1, j=1, \ldots, n \\
& \left(\partial_{y}^{+} u\right)_{i, j} \stackrel{\text { def }}{=} u_{i, j+1}-u_{i, j}, \quad \text { for } i=1, \ldots, n, j=1, \ldots, n-1
\end{aligned}
$$

In addition, to satisfy the Nuemann boundary condition $\frac{\partial u}{\partial n}=0$ on image boundary, the forward differentials on the image right and bottom edges, $\left(\partial_{x}^{+} u\right)_{n, j}$, for $j=1, \ldots, n$, and $\left(\partial_{y}^{+} u\right)_{i, n}$, for $i=1, \ldots, n$, are defined to be zero.

Next we introduce the new variables $t_{i, j}$ and the 3 -dimensional second-order cones

$$
\left(t_{i, j} ;\left(\partial_{x}^{+} u\right)_{i, j},\left(\partial_{y}^{+} u\right)_{i, j}\right) \in \mathcal{K}^{3}
$$

for each pixel $(i, j), i, j=1, \ldots, n$. Each $t_{i, j}$ is no less than $\left(\left(\partial_{x}^{+} u\right)_{i, j}^{2}+\right.$ $\left.\left(\partial_{y}^{+} u\right)_{i, j}^{2}\right)^{1 / 2}$, so minimizing $t_{i, j}$ has the same effect of minimizing $\left(\left(\partial_{x}^{+} u\right)_{i, j}^{2}+\right.$ $\left.\left(\partial_{y}^{+} u\right)_{i, j}^{2}\right)^{1 / 2}$. Therefore, we can express $\min T V(u)$ as $\min \sum_{i, j} t_{i, j}$ subject to the constraints in (19).

Now we are in the position to cast the non- $T V$ fidelity terms in models (5), (8), and (4) as SOCP objectives and constraints.

A piece-wise linear constraint $\int|f-u| \leq \sigma$ in the TV- $L^{1}$ model can be expressed discretely as follows:

$$
\begin{aligned}
\left(f_{i, j}-u_{i, j}\right) & \leq \sigma_{i, j}, \quad \text { for } i, j=1, \ldots, n \\
\left(u_{i, j}-f_{i, j}\right) & \leq \sigma_{i, j}, \quad \text { for } i, j=1, \ldots, n \\
\sum_{i, j} \sigma_{i, j} & \leq \sigma .
\end{aligned}
$$

But if $\int|f-u|$ appears as a minimization objective as is the case in (4), with out loss of generality say the objective is $\min |x|$, one can introduce an extra variable ( $t$ or $s$ below) and transform min $|x|$ into equivalent problems:

$$
\begin{aligned}
& \min |x| \\
& \min \quad t \quad \text { s.t. } \quad x \leq t,-x \leq t \\
(s \stackrel{\text { def }}{=} t+x) & \Longleftrightarrow \min (s-x) \text { s.t. } \quad 2 x \leq s, s \geq 0 .
\end{aligned}
$$


Both Problems (24) and (25) consist of a linear objective and two linear constraints. However, Problem (25) is preferred by some solvers since the nonnegativity constraint $s \geq 0$ is cheaper to handle [28].

For Meyer's model (5), we define the discretized version of $\|v\|_{G}$ as the infimum of

$$
\left\|\sqrt{g_{1}^{2}(i, j)+g_{2}^{2}(i, j)}\right\|_{L^{\infty}}
$$

over all $g_{1}, g_{2} \in \mathbb{R}^{(n+1)^{2}}$ satisfying $v=\partial_{x}^{+} g_{1}+\partial_{y}^{+} g_{2}$ using forward finite differences. To express $\min \|v\|_{G}$ (or, equivalently, $\min \left\|\sqrt{g_{1}^{2}(i, j)+g_{2}^{2}(i, j)}\right\|_{L^{\infty}}$ ) in an SOCP, we introduce a variable $s$ and a 3-dimensional second-order cone

$$
\left(g_{0}(i, j) ; g_{1}(i, j), g_{2}(i, j)\right) \in \mathcal{K}^{3}
$$

for each $i, j$; hence, $\min \|v\|_{G}$ can be equivalently expressed as

$$
\min s, \quad \text { s.t. } g_{0}(i, j) \leq s \text { and }(26) \text {, for all } i, j \text {. }
$$

Next, we present the ways to express the two penalty terms in (8) in SOCPs. Using forward finite difference, the residual penalty term $\int\left|f-u-\partial_{1} g_{1}-\partial_{2} g_{2}\right|^{2}$ is implemented discretely as:

$$
\sum_{i, j}\left|f-u-\partial_{x}^{+} g_{1}-\partial_{y}^{+} g_{2}\right|^{2}
$$

Clearly, minimizing (28) is equivalent to minimizing $s_{1}$ subject to the following chain of constraints:

$$
\begin{aligned}
2 s_{1} s_{2} & \geq s_{3}^{2}, \\
s_{2} & =1 / 2, \\
s_{3} & =s_{4}, \\
s_{4}^{2} & \geq \sum_{1 \leq i, j \leq n} r_{i, j}^{2}, \\
f-u-\partial_{x}^{+} g_{1}-\partial_{y}^{+} g_{2} & =r_{i, j},
\end{aligned}
$$

where (30), (31), and (33) are linear constraints, (29) can be formulated as $\left(s_{1} ; s_{2} ; s_{3}\right) \in \mathcal{Q}^{3}$, and $(32)$ can be formulated as $\left(s_{4} ;\left[r_{i, j}\right]_{1 \leq i, j \leq n}\right) \in \mathcal{K}^{n^{2}+1}$.

For the penalty term

$$
\mu\left[\int\left(\sqrt{g_{1}^{2}+g_{2}^{2}}\right)^{p}\right]^{1 / p}
$$

in (8) there are three cases to consider. When $p=1$, the minimization of (34) can be formulated as the minimization of the sum of $\mu g_{0}(i, j)$ over all $i, j=1, \ldots, n$, where $g_{0}(i, j)$ is subject to (26). When $p=\infty,(34)$ is equal to $\mu\|v\|_{G}$ as in Meyer's model and hence one can solve (27) to minimize (34). 
When $1<p<\infty$, we use second-order cone formulations presented in [1]. Let us study the general case of the $p$-norm inequality

$$
\left(\sum_{i=1}^{n}\left|x_{i}\right|^{l / m}\right)^{m / l} \leq t
$$

where $l / m=p \geq 1$ and $t$ is either a given positive scale or a variable to minimize. If we introduce $s_{i} \geq 0$, for $i=1, \ldots, n$, we can express (35) as the following set of inequalities:

$$
\begin{gathered}
\left|x_{i}\right| \leq s_{i}^{m / l} t^{(l-m) / l}, s_{i} \geq 0, \quad \text { for } i=1, \ldots, n \\
\sum_{i=1}^{n} s_{i} \leq t
\end{gathered}
$$

which is equivalent to

$$
\begin{gathered}
x_{i} \leq s_{i}^{m / l} t^{(l-m) / l}, \quad-x_{i} \leq s_{i}^{m / l} t^{(l-m) / l}, s_{i} \geq 0, \quad \text { for } i=1, \ldots, n \\
\sum_{i=1}^{n} s_{i} \leq t,
\end{gathered}
$$

Let us now illustrate how to express the nontrivial inequality constraints in (38) as a set of 3-dimensional rotated second-order cones and linear inequalities by a concrete example. Suppose $p=5 / 3$, i.e., $m=3, l=5$. Dropping the subscript $i$ and introducing a scalar $z \geq 0$ such that $z+x \geq 0$, it is easy to verify that the first inequality in (38) is equivalent to $z+x \leq s^{3 / 5} t^{2 / 5}, z \geq 0$ and $z+x \geq 0$, which in turn is equivalent to $(z+x)^{8} \leq s^{3} t^{2}(z+x)^{3}, z \geq 0$ and $z+x \geq 0$. The latter can be further expressed as the following system of inequalities:

$$
\begin{aligned}
& w_{1}^{2} \leq s(z+x), \quad w_{2}^{2} \leq w_{1} s, \quad w_{3}^{2} \leq t(z+x) \\
& (z+x)^{2} \leq w_{2} w_{3}, \quad z \geq 0, \quad z+x \geq 0
\end{aligned}
$$

where the first four inequalities form four rotated second-order cones. The same argument applies to $-x \leq s^{3 / 5} t^{2 / 5}$ if we replace $x$ wherever it appears in the argument by $-x$.

\subsection{SOCP model formulations}

We now combine the SOCP expressions derived in the last subsection to give complete SOCP formulations for (4), (5), and (8). 


\subsubsection{The $T V-L^{1}$ model}

Below we give both the SOCP formulations of the constraint and Lagrangian (differences indicated in parenthesis) versions (3) and (4) of the TV- $L^{1}$ model:

$$
\begin{aligned}
& \min \sum_{1 \leq i, j \leq n} t_{i, j} \quad \text { (Lagrangian ver.: } \sum_{1 \leq i, j \leq n} t_{i, j}+\lambda s \text { ) } \\
& \text { s.t. }-\left(\partial_{x}^{+} u\right)_{i, j}+\left(u_{i+1, j}-u_{i, j}\right)=0 \text {, } \\
& \text { for } i, j=1, \ldots, n \\
& -\left(\partial_{y}^{+} u\right)_{i, j}+\left(u_{i, j+1}-u_{i, j}\right)=0, \\
& \text { for } i, j=1, \ldots, n \\
& u_{i, j}+\sigma_{i, j} \geq f_{i, j}, \\
& \text { for } i, j=1, \ldots, n \\
& u_{i, j}-\sigma_{i, j} \leq f_{i, j}, \\
& \text { for } i, j=1, \ldots, n \\
& \sum_{i, j} \sigma_{i, j} \leq \sigma, \quad \text { (Lagrangian ver.: replace } \sigma \text { by } s \text { ) } \\
& \left(t_{i, j} ;\left(\partial_{x}^{+} u\right)_{i, j},\left(\partial_{y}^{+} u\right)_{i, j}\right) \in \mathcal{K}^{3} \\
& \text { for } i, j=1, \ldots, n \text {, }
\end{aligned}
$$

where $u, \partial_{x}^{+} u, \partial_{y}^{+} u, \sigma_{i, j}, s$, and $t$ are variables and $f, \lambda$, and $\sigma$ are constants.

Moreover, to solve the TV- $L^{1}$ model with Neumann boundary conditions, we include the additional constraints

$$
\begin{aligned}
& \left(\partial_{x}^{+} u\right)_{n, j}=0, \text { for } j=1, \ldots, n \\
& \left(\partial_{y}^{+} u\right)_{i, n}=0, \text { for } i=1, \ldots, n
\end{aligned}
$$

in (42) since the boundary constraints containing them also contain $u_{n+1, j}$ 's and $u_{i, n+1}$ 's, which are out of the image domain and undefined. This convention also applies to the SOCP formulations of the other two TV-based models below.

\subsubsection{Meyer's model}

The following is the SOCP formulation for the constraint version (5) of Meyer's model (the differences of the one for the Lagrangian version (6) are given below 
in parenthesis).

$$
\left.\min \sum_{1 \leq i, j \leq n} t_{i, j} \text { (Lagrangian ver.: } \sum_{1 \leq i, j \leq n} t_{i, j}+\lambda s\right)
$$

$$
\begin{aligned}
& \text { s.t. } u_{i, j}+v_{i, j}=f_{i, j}, \\
& -\left(\partial_{x}^{+} u\right)_{i, j}+\left(u_{i+1, j}-u_{i, j}\right)=0, \\
& -\left(\partial_{y}^{+} u\right)_{i, j}+\left(u_{i, j+1}-u_{i, j}\right)=0, \\
& v_{i, j}-\left(g_{1, i+1, j}-g_{1, i, j}+g_{2, i, j+1}-g_{2, i, j}\right)=0 \text {, } \\
& \left.g_{0, i, j} \leq \sigma, \quad \text { (Lagrangian ver.: } g_{0, i, j} \leq s\right) \\
& \left(t_{i, j} ;\left(\partial_{x}^{+} u\right)_{i, j} ;\left(\partial_{y}^{+} u\right)_{i, j}\right) \in \mathcal{K}^{3}, \\
& \left(g_{0, i, j} ; g_{1, i, j}, g_{2, i, j}\right) \in \mathcal{K}^{3} \text {, }
\end{aligned}
$$

where $u, v, \partial_{x}^{+} u, \partial_{y}^{+} u, g_{0}, g_{1}, g_{2}, t$, and $s$ are variables and $f, \sigma$, and $\lambda$ are constants. Although solving for $u$ and $v$ is our ultimate goal, they can be eliminated from the above formulation using the second, third, and fourth sets of equations in (43). After solving the resulting problem for the remaining variables, $v$ can be recovered from $\left(g_{1, i+1, j}-g_{1, i, j}+g_{2, i, j+1}-g_{2, i, j}\right)$ and $u$ from $f-v$.

\subsubsection{The Vese-Osher (VO) model}

The Vese-Osher model [35] with $p=1$ is

$$
\inf _{u, g_{1}, g_{2}}\left\{\int|\nabla u| d x+\lambda \int\left|f-u-\partial_{1} g_{1}-\partial_{2} g_{2}\right|^{2} d x+\mu \int\left|\sqrt{g_{1}^{2}+g_{2}^{2}}\right| d x\right\} .
$$

In this model the authors relax the constraint $f-u=\partial_{1} g_{1}-\partial_{2} g_{2}$ by penalizing the square of its violation (the second term in (44)) because they can then write down the Euler-Lagrange equations of (44) and use the gradient descent method to find a solution. Ideally, $\lambda=\infty$ should be used when solving (44) to give a decomposition of $f$ into $u$ and $v$ with no residual. This is equivalent to solving the residual-free version (45) below. Although we can formulate both (44) and (45) as SOCPs, we chose to solve the latter in our numerical tests because using a large $\lambda$ in (44) makes it difficult to numerically solve its SOCP accurately.

The constraint version of the Vese-Osher model is

$$
\inf _{u, g_{1}, g_{2}}\left\{\int|\nabla u| \mathrm{d} x+\mu \int\left|\sqrt{g_{1}^{2}+g_{2}^{2}}\right| \mathrm{d} x, \quad \text { s.t. } f-u=\partial_{1} g_{1}+\partial_{2} g_{2}\right\},
$$


which has a SOCP formulation as

$$
\begin{aligned}
& \min \sum_{1 \leq i, j \leq n} t_{i, j}+\mu \sum_{1 \leq i, j \leq n+1}\left(w_{i, j}-g_{0, i, j}\right) \\
& \text { s.t. } u_{i, j}+v_{i, j}=f_{i, j}, \quad \text { for } i, j=1, \ldots, n \\
& -\left(\partial_{x}^{+} u\right)_{i, j}+\left(u_{i+1, j}-u_{i, j}\right)=0, \quad \text { for } i, j=1, \ldots, n \\
& -\left(\partial_{y}^{+} u\right)_{i, j}+\left(u_{i, j+1}-u_{i, j}\right)=0, \quad \text { for } i, j=1, \ldots, n \\
& v_{i, j}-\left(g_{1, i+1, j}-g_{1, i, j}+g_{2, i, j+1}-g_{2, i, j}\right)=0, \text { for } i, j=1, \ldots, n \\
& 2 g_{0, i, j} \leq w_{i, j}, w_{i, j} \geq 0, \quad \text { for } i, j=1, \ldots, n+1 \\
& \left(t_{i, j} ;\left(\partial_{x}^{+} u\right)_{i, j} ;\left(\partial_{y}^{+} u\right)_{i, j}\right) \in \mathcal{K}^{3}, \quad \text { for } i, j=1, \ldots, n \\
& \left(g_{0, i, j} ; g_{1, i, j}, g_{2, i, j}\right) \in \mathcal{K}^{3}, \quad \text { for } i, j=1, \ldots, n+1 \text {, }
\end{aligned}
$$

where $u, v, \partial_{x}^{+} u, \partial_{y}^{+} u, g_{0}, g_{1}, g_{2}, t$, and $w$ are variables and $f$ and $\mu$ are constants. Similar to the SOCP for Meyer's model, $u$ and $v$ can be eliminated from the above formulation using the first, second, third, and fourth sets of equations in (46) and recovered from remaining variables after solving the problem.

The SOCPs for $p=l / m>1$ and $p=\infty$ can be derived using the techniques discussed in the last subsection.

\section{Numerical results}

In this section, we present numerical results for the three cartoon-texture decomposition models and compare them. In all cases we solved the Lagrangian version (4) of the TV- $L^{1}$ model, the constraint version (5) of Meyer's model, and the residual-free version (45) of the Vese-Osher (VO) model (8) with $p=1$.

We used the commercial optimization package Mosek [28] as our SOCP solver. Mosek is designed to solve a variety of large-scale optimization problems, including SOCPs. Before solving a large-scale problem, Mosek uses a presolver to remove redundant constraints and variables and reorders constraints to speed up the numerical linear algebra required by the interior-point SOCP algorithm that it uses. We could also have designed dedicated reordering algorithms for each of the three models following an approach similar to the one described in [19] to lower solution times. However, this was not done as our focus was on decomposition quality comparisons. 
In order to obtain accurate solutions for comparisons, we specified tolerances of 1.0e- 8 for all maximum primal and dual equation infeasibilities and significant digit requirements. In a couple of experiments, Mosek terminated and returned a solution that did not satisfy these tolerances when numerical difficulties prevented the solver from improving the solution accuracy. However, all returned solutions were accurate enough for our purpose of comparisons. We report in Table 1 the measures of maximum primal and dual equation infeasibilities, maximum primal and dual bound infeasibilities, and duality gaps of the solutions returned by Mosek in all of the 2D experiments. The $1 \mathrm{D}$ problems were much smaller and easier than the 2D's, so we obtained solutions with even higher accuracies. The two instances of small and negative duality gaps were due to numerical inaccuracies in Mosek. Nevertheless, all of the minimum objective values had at least five accurate digits as shown by the powers of the significant digits reported in Table 1.

In our first set of tests, we applied the models to noise-free inputs: a 1D signal and three $2 \mathrm{D}$ images.

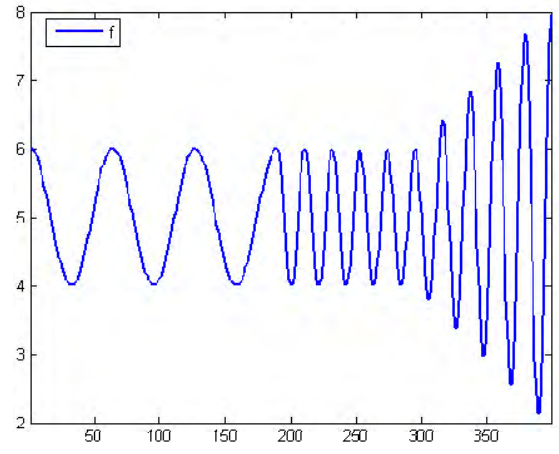

(a) Original

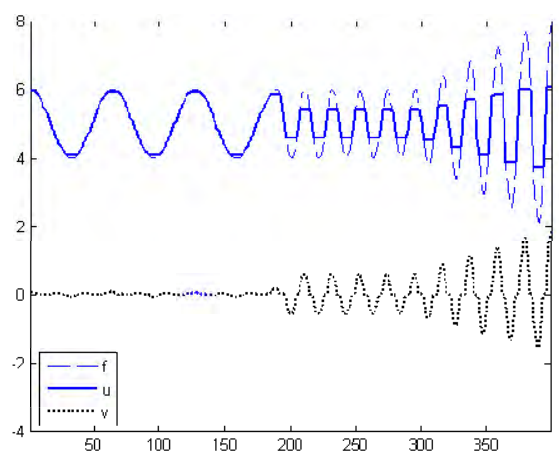

(c) $\mathrm{TV}-L^{1}(\lambda=0.25)$

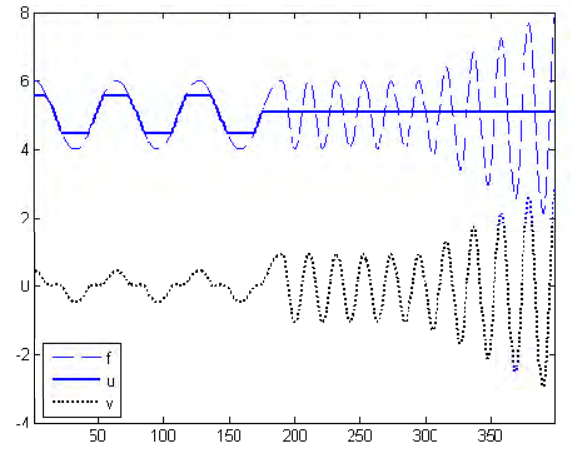

(b) $\mathrm{TV}-L^{1}(\lambda=0.1)$

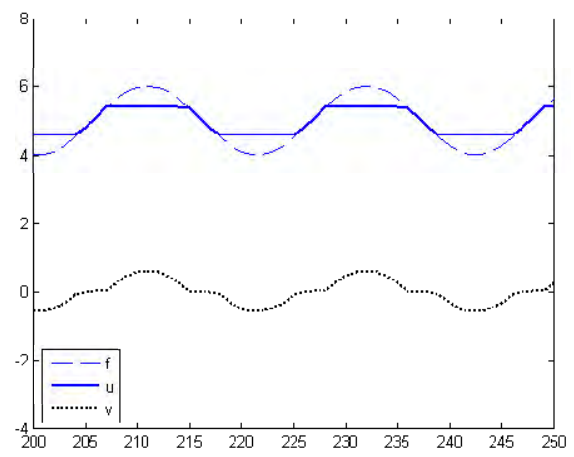

(d) The enlargement of (c) at $i=200, \ldots, 250$

Fig. 1. Example 1: 1D signal decomposition

Example 1: In this test we applied the three models to a 399-point 1D oscil- 
lating signal as depicted in Figure 1 (a). The signal generated was:

$$
f_{i}=5+ \begin{cases}\cos \left(\frac{63 \pi}{2} i\right), & i=1, \ldots, 189 \\ \cos \left(\frac{21 \pi}{2} i\right), & i=190, \ldots, 294 \\ \left(1+\frac{(i-295)}{50}\right) \cos \left(\frac{21 \pi}{2} i\right), & i=295, \ldots, 399\end{cases}
$$

This signal is a shifted (by +5 ) sample of an oscillating function consisting of three sections: the first and second sections contain three and five cosine cycles with wavelengths 63 and 21, respectively, where the second triples the frequency of the first; the third section is a duplicate of the second with linearly increasing amplifications from $1 \times$ to about $3 \times$. We applied the three models to this signal each with two choices of their perspective parameters: $\lambda, \sigma$, and $\mu$. The parameter values used to obtain all results are indicated below the $u$ and $v$ signals and images. The first of each parameter was chosen to be small enough or large enough to remove the second section completely from $u$; the second value chosen only partially removed the second section from $u$. We were interested in learning how the three models performed on the signal in the first and third sections. In Figures 1 (b)-(d) and 2 (e)-(j), we present the decomposed signals $u$ and $v$, along with the input $f$. In these plots $f, u$, and $v$ are plotted by dashed, solid, and dotted curves, respectively. For more detailed views, the same parts, $[200,250]$ on the $x$-axis, of Plots (c), (f), and (i) are horizontally enlarged by eight times and shown as Plots (d), (g), and (j), respectively.

The TV- $L^{1}$ model decomposed the signal by the scale (i.e., the width in 1D) of the super and lower level sets of the signal, independent of wave amplitude. In general, any parts of the signal with level sets of widths less than $2 / \lambda$ were removed from $u$ according to the results from $[2,38]$. With $\lambda=0.1$, the complete second and third sections of the signal were excluded from $u$ in Figure 1 (b) since their half wavelength, equal to the width of the super and lower level sets at 5 which is $21 / 2=10.5$, was less than $2 / \lambda=20$. In contrast for $\lambda=0.25$ as shown in Figures 1 (c) and (d), since $2 / \lambda=8$ was smaller than the half wavelength $(=10.5)$, only parts of the signal were not in $u$. Moreover, we can see from Figure 1 (d) that those parts that were chopped off from $u$ had spans with width equal to $2 / \lambda=8$.

The Meyer and Vese-Osher (VO) models did not decompose $f$ this way. Meyer's model tended to capture the pattern of the oscillations in $v$, rather than the oscillations themselves. As depicted in Figure 2 (e), Meyer's model with $\sigma=5.0$ gave a $v$ that had waves with more uniform amplitudes than those of the $v$ generated by the TV- $L^{1}$ model depicted in Figure 1 (b). Correspondingly, the $u$ of Meyer's model clearly compensated for the increasing amplification of the signal in the third section and did not completely vanish in both the second and third sections as shown again in Figure 2 (e). With both $\sigma=5.0$ and 1.5, Meyer's model resulted in artifacts that we do not 


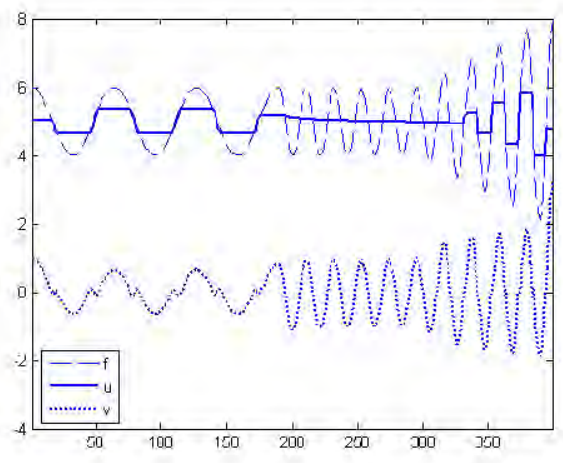

(e) Meyer $(\sigma=5.0)$

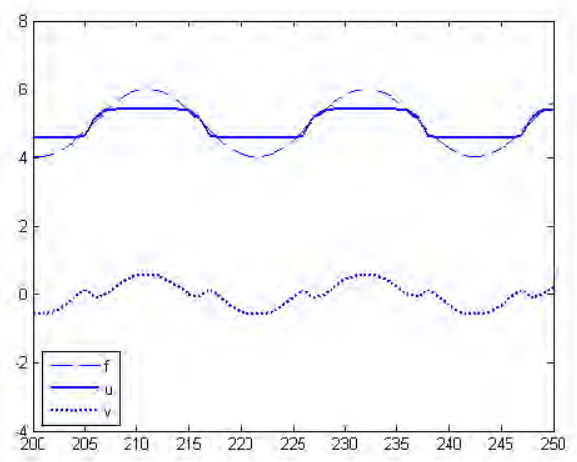

(g) The enlargement of (f) at $i=200, \ldots, 250$

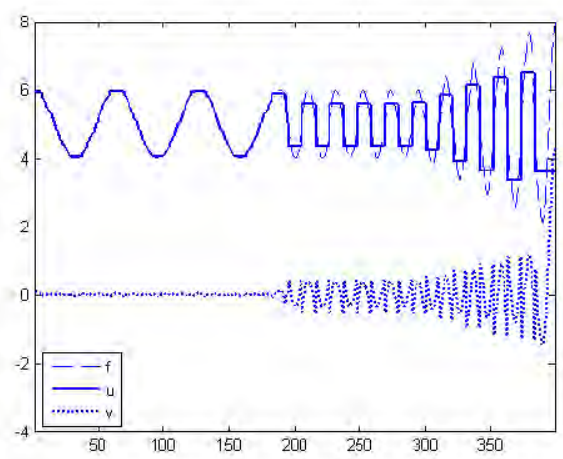

(i) $\operatorname{VO}(\mu=0.075)$

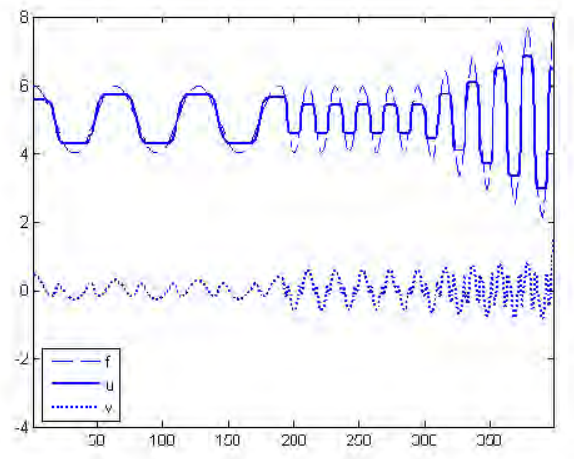

(f) Meyer $(\sigma=1.5)$

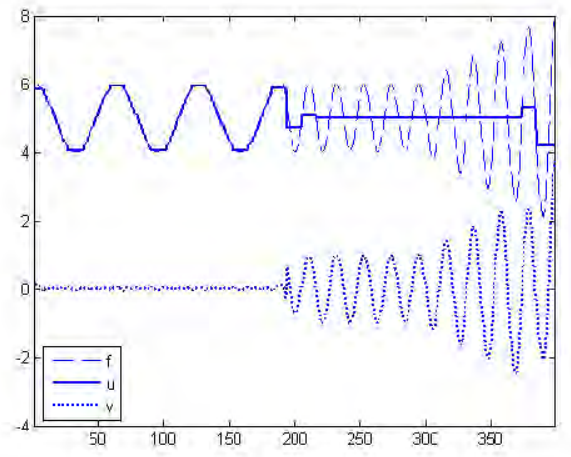

(h) $\mathrm{VO}(\mu=0.06)$

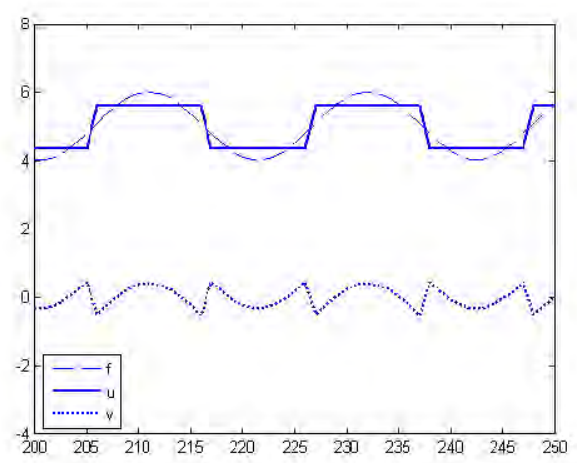

(j) The enlargement of (i) at $i=200, \ldots, 250$

Fig. 2. Example 1: 1D signal decomposition (continue)

know how to relate to $f$. In Figures 2 (e)-(g), we can find that, in addition to chopping off the waves, the model created the nonconforming $u$ 's, which had curves that do not match those in $f$. In $v$, we can observe these artifacts more clearly as highlighted in Plot (g). Intuitively, we interpret this phenomenon as the tendency of Meyer's model to create oscillations in $v=\operatorname{div}(\vec{g})$.

Similar artifacts can also be found in the results Figures $2(\mathrm{~h})-(\mathrm{j})$ of the VO model, but the differences are that the VO model generated $u$ 's that have a block-like structure and thus $v$ 's with more complicated patterns. However, the 
Table 1

Mosek termination measures

\begin{tabular}{|c|c|c|c|c|c|}
\hline Input & Model & Primal Eq/Bnd Infeas. & Dual Eq/Bnd Infeas. & Duality Gap & Sig. Digits \\
\hline \multirow{3}{*}{ Finger } & $\mathrm{TV}-L^{1}$ & $2.84 \mathrm{e}-14 / 8.21 \mathrm{e}-7$ & $4.96 \mathrm{e}-8 / 0 \mathrm{e} 0$ & $9.74 \mathrm{e}-4$ & $6.38 \mathrm{e}-9$ \\
\hline & Meyer & $5.24 \mathrm{e}-14 / 4.26 \mathrm{e}-6$ & $3.22 \mathrm{e}-7 / 0 \mathrm{e} 0$ & $1.62 \mathrm{e}-1$ & $4.78 \mathrm{e}-6$ \\
\hline & $\mathrm{VO}$ & $6.03 \mathrm{e}-07 / 1.10 \mathrm{e}-5$ & $1.42 \mathrm{e}-7 / 0 \mathrm{e} 0$ & $1.95 \mathrm{e}-3$ & $3.56 \mathrm{e}-8$ \\
\hline \multirow{3}{*}{$\begin{array}{l}\text { Barbara } \\
\text { (part) }\end{array}$} & TV $-L^{1}$ & $5.68 \mathrm{e}-14 / 2.37 \mathrm{e}-5$ & $4.63 \mathrm{e}-9 / 0 \mathrm{e} 0$ & $-2.09 \mathrm{e}-4$ & $-3.27 \mathrm{e}-10$ \\
\hline & Meyer & $5.68 \mathrm{e}-14 / 8.11 \mathrm{e}-6$ & $1.10 \mathrm{e}-7 / 0 \mathrm{e} 0$ & $1.16 \mathrm{e}-1$ & $4.40 \mathrm{e}-7$ \\
\hline & $\mathrm{VO}$ & $3.54 \mathrm{e}-05 / 6.09 \mathrm{e}-5$ & $4.81 \mathrm{e}-9 / 0 \mathrm{e} 0$ & $-4.54 \mathrm{e}-3$ & $-1.22 \mathrm{e}-8$ \\
\hline \multirow{3}{*}{$\begin{array}{l}\text { 4tex- } \\
\text { ture }\end{array}$} & TV- $L^{1}$ & $5.68 \mathrm{e}-14 / 1.98 \mathrm{e}-6$ & $1.05 \mathrm{e}-7 / 0 \mathrm{e} 0$ & $1.31 \mathrm{e}-2$ & $7.54 \mathrm{e}-9$ \\
\hline & Meyer & $6.75 \mathrm{e}-14 / 5.08 \mathrm{e}-6$ & $4.04 \mathrm{e}-7 / 0 \mathrm{e} 0$ & $1.36 \mathrm{e} 00$ & $4.96 \mathrm{e}-6$ \\
\hline & $\mathrm{VO}$ & $2.58 \mathrm{e}-06 / 2.74 \mathrm{e}-6$ & $1.34 \mathrm{e}-7 / 0 \mathrm{e} 0$ & $7.87 \mathrm{e}-3$ & $4.76 \mathrm{e}-9$ \\
\hline noisy & TV- $L^{1}$ & $5.68 \mathrm{e}-14 / 1.25 \mathrm{e}-6$ & $6.22 \mathrm{e}-8 / 0 \mathrm{e} 0$ & $7.46 \mathrm{e}-3$ & $6.45 \mathrm{e}-9$ \\
\hline \multirow{2}{*}{$\begin{array}{l}\text { Barbara } \\
\text { (part) }\end{array}$} & Meyer & $6.75 \mathrm{e}-14 / 3.74 \mathrm{e}-6$ & $2.36 \mathrm{e}-7 / 0 \mathrm{e} 0$ & $3.28 \mathrm{e}-1$ & $1.22 \mathrm{e}-6$ \\
\hline & $\mathrm{VO}$ & $6.75 \mathrm{e}-05 / 9.87 \mathrm{e}-5$ & $1.55 \mathrm{e}-9 / 0 \mathrm{e} 0$ & $2.38 \mathrm{e}-3$ & $4.16 \mathrm{e}-9$ \\
\hline
\end{tabular}

VO model with $\mu=0.06$ gave a $u$ and $v$ quite similar to the $u$ and $v$ produced by the TV- $L^{1}$ model with $\lambda=0.25$. In Figure $2(\mathrm{~h})$, most of the signal in the second and third section was extracted from $u$, leaving very little signal near the boundary of these signal parts. In short, the VO model performed like an approximation of Meyer's model but with certain features closer to those of the TV- $L^{1}$ model.

Example 2: In this test we applied the three models to a $117 \times 117$ fingerprint as depicted in Figure 3 (a). This fingerprint has slightly inhomogeneous brightness because the background near the center of the finger is whiter than the rest. We believe that the inhomogeneity like this is not helpful to the recognition and comparison of fingerprints so should better be corrected. Figures 4 (a), (b), and (c) depict the decomposition results given by applying Meyer's, the VO, and the TV- $L^{1}$ models, respectively. The left half of each figure gives the cartoon part $u$, and the right half gives the texture part $v$, which is more important for recognition. Since the VO model is an approximation to Meyer's model, they gave very similar results. We can observe in Figures 4 (a) and (b) that their cartoon parts are close to each other, but slightly different from the cartoon in Figure 4 (c). The texture parts of (a) and (b) appear to be more homogenous than that of $(\mathrm{c})$, which shows the whiter background near the center of the finger. However, in (c) edges are sharper than they are in the other two figures. Although no fingerprint recognition was done using the resulting images, we conclude that the Meyer and VO models seem to give more useful results than the TV- $L^{1}$ model in this experiment.

Example 3: We tested textile texture decomposition by applying the three models to a part of the image "Barbara" as depicted in Figure 3 (c). The 


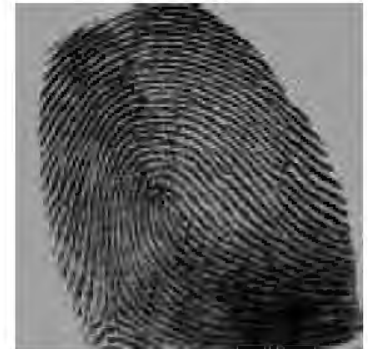

(a)

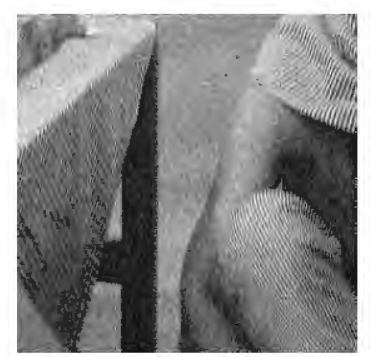

(d)

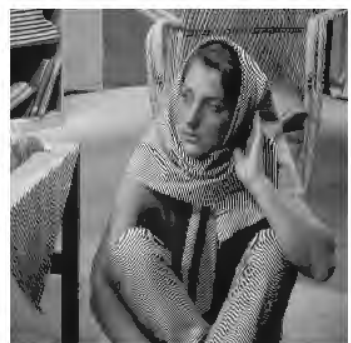

(b)

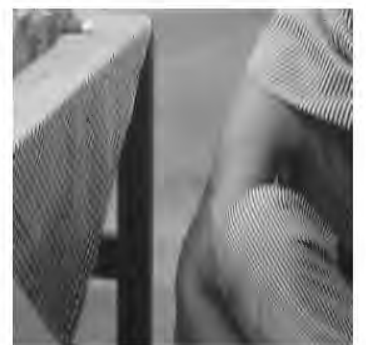

(c)

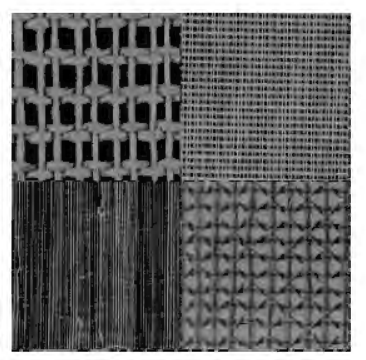

(e)

Fig. 3. Inputs: (a) original $117 \times 117$ fingerprint, (b) original $512 \times 512$ Barbara, (c) a $256 \times 256$ part of original Barbara, (d) a $256 \times 256$ part of noisy Barbara (std. $=20$ ), (e) original $256 \times 2564$ texture.

full Barbara image is depicted in Figure 3 (b). Ideally, only the table texture and the strips on Barbara's clothes should be extracted. Surprisingly, Meyer's model did not give good results in this test. In Figure 4 (d) and (e) we can observe that the texture $v$ 's contain inhomogeneous background, depicting Barbara's right arm and the table leg. To illustrate this effect, we used two different $\sigma$ 's, one very conservative and then one aggressive - namely, a small $\sigma$ and then a large $\sigma$ - in Meyer's model. The outputs are depicted in Figure 4 (d) and (e), respectively. With a small $\sigma=6$, most of the table cloth and clothes textures remained in the cartoon $u$ part. However, while a large $\sigma=15$ allowed Meyer's model to remove more textures from $u$, it also allowed $u$ to contain lots of unwanted inhomogeneous background as mentioned above. One can imagine that by further increasing $\sigma$ we would get a result with less texture left in the $u$ part, but with more inhomogeneous background left in the $v$ part. While Meyer's method gave unsatisfactory results, the other two models gave very good results in this test as little background appears in the $v$ parts of Figures 4 (f) and (g). The TV- $L^{1}$ model still generated a little sharper cartoon than the VO model in this test. The biggest difference, however, is that the $\mathrm{TV}-L^{1}$ model kept most brightness changes in the texture part while the VO model kept more such changes in the cartoon part. In the top right regions of the output images, the wrinkles of Barbara's clothes are shown in the $u$ part of Figure $4(\mathrm{~g})$ but in the $v$ part of $(\mathrm{g})$. This shows that the texture extracted by the TV- $L^{1}$ has a wider dynamic range. In this experiments, the VO and the TV- $L^{1}$ models gave us more satisfactory results than Meyer's model.

Example 4: We applied the three decompositions models to 4-textures de- 


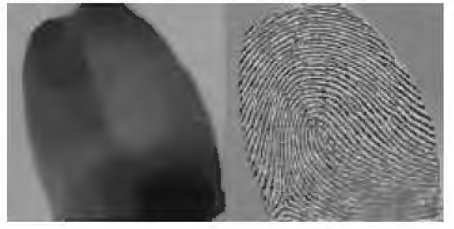

(a) Meyer $(\sigma=35)$

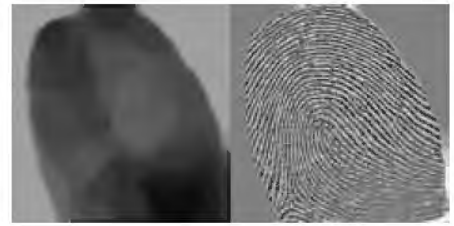

(b) $\mathrm{VO}(\mu=0.1)$

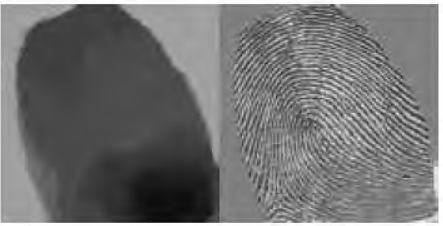

(c) $\operatorname{TV}-L^{1}(\lambda=0.4)$

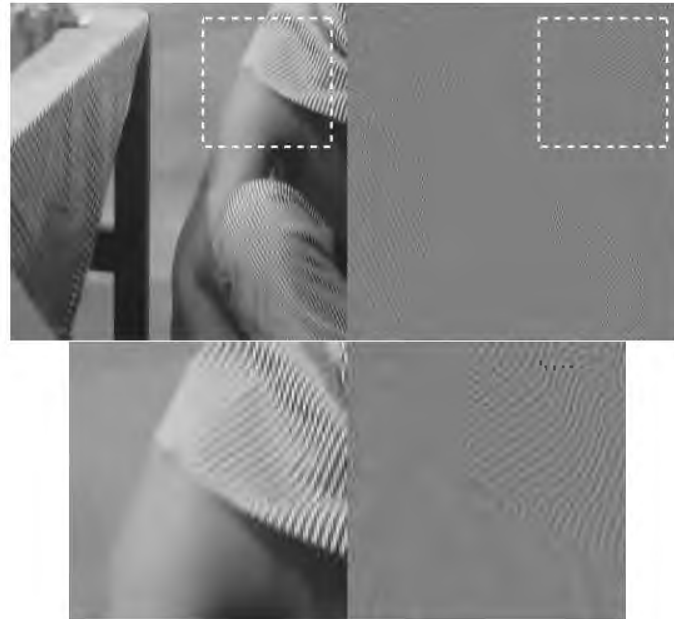

(d) Meyer $(\sigma=6)$. Upper: the $256 \times 256 u$ and $v$; Lower: a zoomed in part of the upper
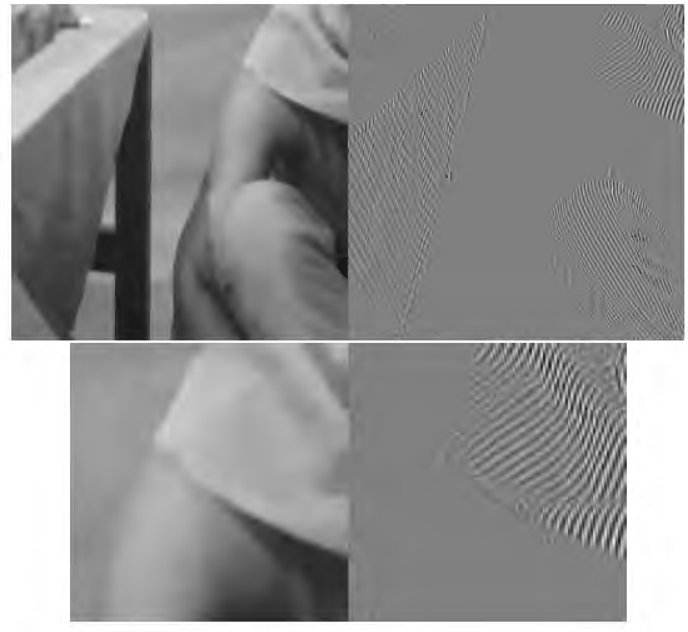

(f) $\mathrm{VO}(\mu=0.5)$. Upper: the $256 \times 256 u$ and $v$; Lower: a zoomed in part of the upper

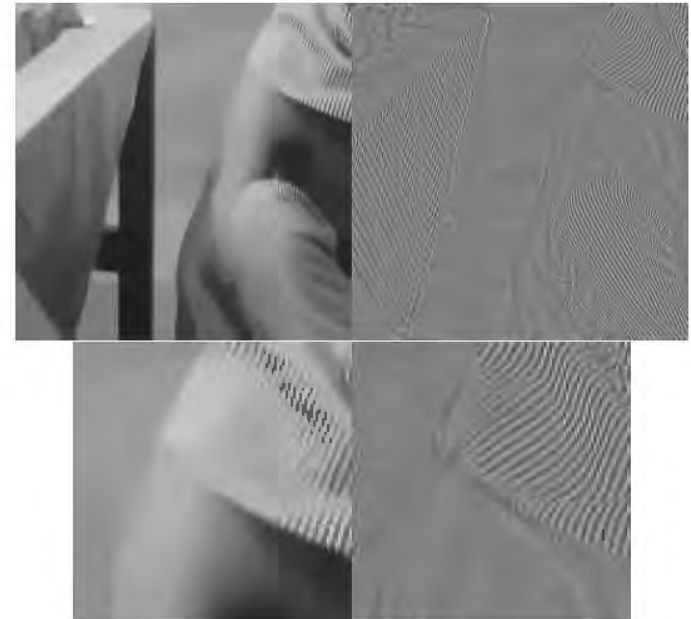

(e) Meyer $(\sigma=15)$. Upper: the $256 \times 256 u$ and $v$; Lower: a zoomed in part of the upper
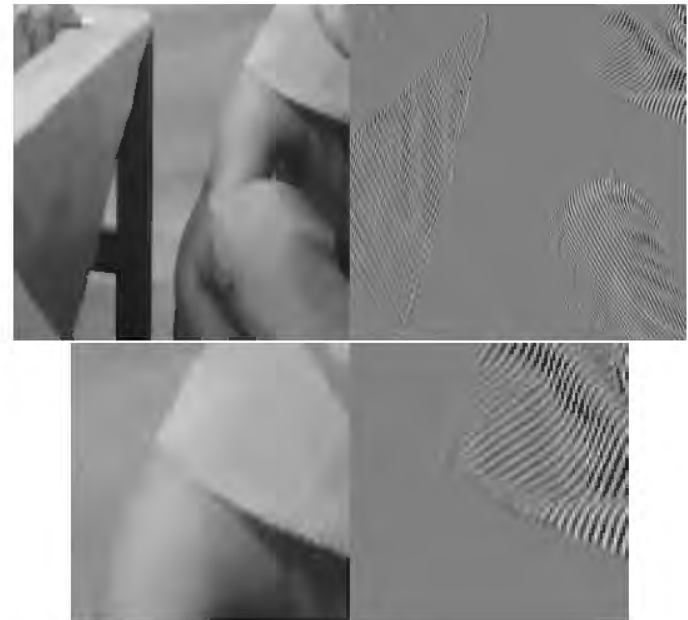

(g) $\mathrm{TV}-L^{1}(\lambda=0.8)$. Upper: the $256 \times 256 u$ and $v$; Lower: a zoomed in part of the upper

Fig. 4. Examples 2 and 3, cartoon-texture decomposition results: left halves - cartoon, right halves - texture.

picted in Figure 3 (e) using parameter values just small enough or large enough for the woven texture (the upper right part) to be extracted to $v$. Figures 5 (a)-(c) demonstrate the differences described in last paragraph more clearly. Both the Meyer and the VO models kept the background brightness changes in the upper right part in $u$ but the TV- $L^{1}$ model did not. However, on the upper left and bottom right parts, the VO model and the TV- $L^{1}$ models behaved similarly while only Meyer's model extracted the pattern of the rope knots in 
$v$. However, the three models decomposed the wood texture (the bottom left part) more or less indistinguishably except that the TV- $L^{1}$ model gave the texture part (i.e., the $v$ part) with stronger contrast as depicted in (i). Once again, the TV- $L^{1}$ model decomposed textures by thresholding on the scales of their level sets, and decomposition by Meyer's model emphasized more the patterns of the textures rather than the textures themselves. The VO model exhibited properties lying in between the two others.

In our second set of tests, we applied the three models to noisy images to see how their decompositions were affected by noise.

Example 5: We applied the three models to the image "Barbara" after adding a substantial amount of Gaussian noise (standard deviation equal to 20). The resulting noisy image is depicted in Figure $3(\mathrm{~d})$. All three models removed the noise together with the texture from $f$, but noticeably, the cartoon parts $u$ in these results (Figure 5 (d)-(f)) exhibit different degrees of staircasing. Compared to the parameters used in the three models for decomposing noiseless images in Example 3, the parameters used in the Meyer and VO models in this set of tests were changed due to the increase in the $G$-norm of the texture/noise part $v$ that resulted from adding noise. However, we did not change $\lambda$ when applying the TV- $L^{1}$ model since the noise does not change the scales of the feature level sets significantly. In subsequent tests, we used an increased Lagrange multiplier $\mu$ and $\lambda$ when applying the VO and the TV- $L^{1}$ models and a decreased constraint bound $\sigma$ when applying Meyer's model in order to keep the cartoon output $u$ closer to $f$. Nevertheless, the staircase effect remained in the resulting $u$ parts, while noise was not fully removed. To summarize, none of the three decomposition models was able to separate image texture and noise, and in fact all of them exhibited the staircase effect in the presence of noise, well known to occur when minimizing total variation.

The codes used to generate the above results can be downloaded from the first author's homepage.

Finally, an anonymous referee brought to our attention that the paper [8] by Aujol and Chambolle compares the $G$-norm with the $E$-norm (i.e., $\dot{B}_{\infty, \infty}^{-1}$ ) for noise removal and concludes that the latter gives better results.

\section{Conclusion}

In this work, we have computationally studied three total variation based models with discrete inputs: the Meyer, VO, and TV- $L^{1}$ models. We showed how to formulate these models as second-order cone programs, which we solved by an interior-point method. We tested these models using a variety of $1 \mathrm{D}$ sig- 


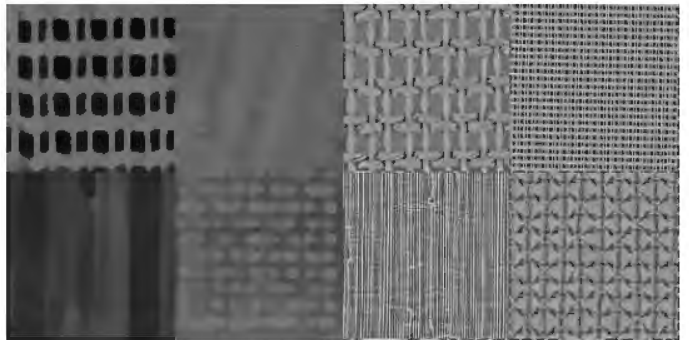

(a) Meyer $(\sigma=50)$

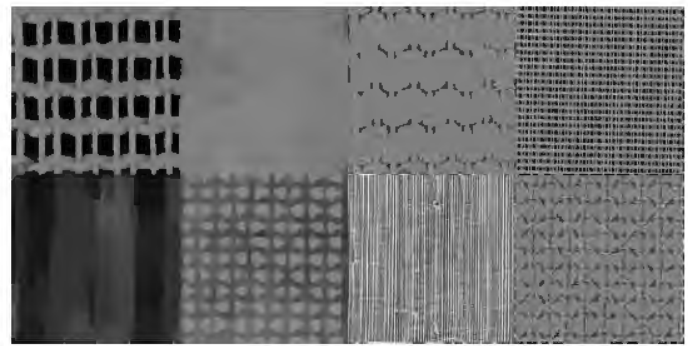

(c) $\mathrm{TV}-L^{1}(\lambda=0.8)$

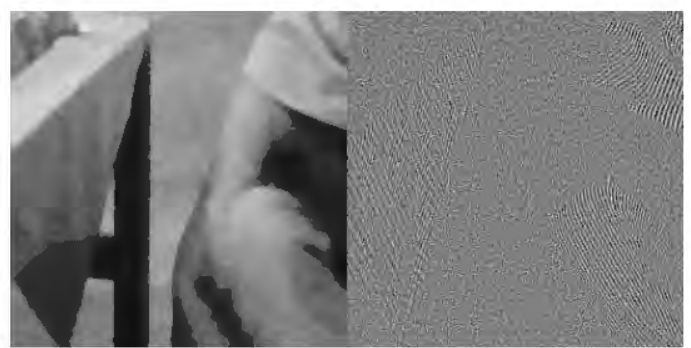

(e) $\operatorname{VO}(\mu=0.5)$

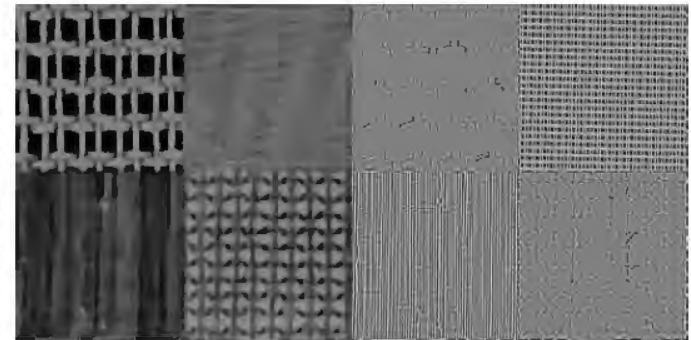

(b) $\mathrm{VO}(\mu=1.0)$

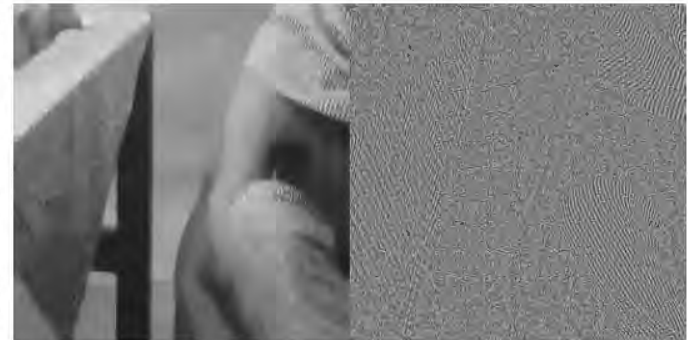

(d) Meyer $(\sigma=20)$

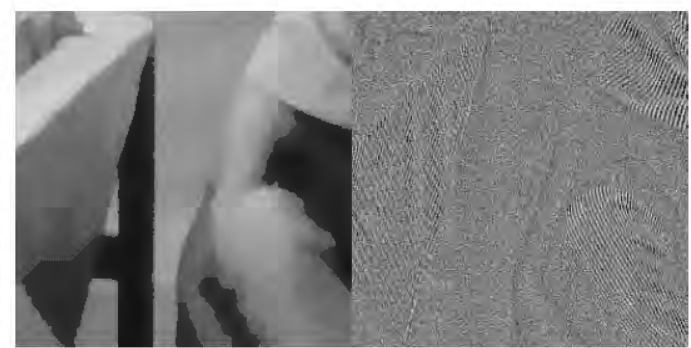

(f) $\mathrm{TV}-L^{1}(\lambda=0.8)$

Fig. 5. Example 4 and 5, cartoon-texture decomposition: left halves - cartoon, right halves - texture/noise.

nals and 2D images to reveal their differences in decomposing inputs into their cartoon and oscillating/small-scale/texture parts. The Meyer model tends to capture the pattern of the oscillations in the input, which makes it well-suited to applications such as fingerprint image processing. On the other hand, the $\mathrm{TV}-L^{1}$ model decomposes the input into two parts according to the geometric scales of the components in the input, independent of the signal intensities, one part containing large-scale components and the other containing smallscale ones. These results agree with those in [9], which compares the ROF, Meyer, and TV- $L^{1}$ models. Our experiments also show that the properties of the VO model fall in between the other two models.

\section{References}

[1] F. Alizadeh, D. Goldfarb, Second-order cone programming, Mathematical Programming 95 (1) (2003) 3-51.

[2] S. Alliney, Digital filters as absolute norm regularizers, IEEE Transactions on 
Signal Processing 40 (6) (1992) 1548-1562.

[3] S. Alliney, Recursive median filters of increasing order: a variational approach, IEEE Transactions on Signal Processing 44 (6) (1996) 1346-1354.

[4] S. Alliney, A property of the minimum vectors of a regularizing functional defined by means of the absolute norm, IEEE Transactions on Signal Processing 45 (4) (1997) 913-917.

[5] G. Aubert, J. F. Aujol, Modeling very oscillating signals, application to image processing, Applied Mathematics and Optimization 51 (2) (2005) 163-182.

[6] J. F. Aujol, G. Aubert, L. Blanc-Feraud, A. Chambolle, Decomposing an image: application to textured images and SAR images, in: Scale-Space'03, Vol. 2695 of Lecture Notes in Computer Science, Springer, 2003.

[7] J. F. Aujol, G. Aubert, L. Blanc-Feraud, A. Chambolle, Image decomposition into a bounded variation component and an oscillating component, Journal of Mathematical Imaging and Vision 22 (1) (2005) 71-88.

[8] J. F. Aujol, A. Chambolle, Dual norms and image decomposition models, International Journal of Computer Vision 63 (1) (2005) 85-104.

[9] J. F. Aujol, G. Gilboa, T. F. Chan, S. Osher, Structure-texture image decomposition - modeling, algorithms, and parameter selection, International Journal of Computer Vision 67 (1) (2006) 111-136.

[10] A. Chambolle, An algorithm for total variation minimization and applications, Journal of Mathematical Imaging and Vision 20 (2004) 89-97.

[11] A. Chambolle, Total variation minimization and a class of binary MRF models, Tech. Rep. UMR CNRS 7641, Ecole Polytechnique (2005).

[12] T. F. Chan, S. Esedoglu, Aspects of total variation regularized $L^{1}$ function approximation, SIAM Journal on Applied Mathematics 65 (5) (2005) 18171837.

[13] T. Chen, T. Huang, W. Yin, X. S. Zhou, A new coarse-to-fine framework for 3D brain MR image registration, in: Computer Vision for Biomedical Image, Vol. 3765 of Lecture Notes in Computer Science, Springer, 2005, pp. 114-124.

[14] T. Chen, W. Yin, X. S. Zhou, D. Domaniciu, T. Huang, Illumination normalization for face recognition and uneven background correction using total variation based image models, in: 2005 IEEE Computer Society Conference on Computer Vision and Pattern Recognition (CVPR'05), Vol. 2, San Diego, 2005, pp. $532-539$.

[15] T. Chen, W. Yin, X. S. Zhou, D. Comaniciu, T. Huang, Total variation models for variable lighting face recognition, IEEE Transactions of Pattern Analysis and Machine Intelligence (PAMI) 28 (9) (2006) 1519-1524.

[16] G. Chung, T. Le, L. Lieu, N. Tanushev, L. Vese, Computational methods for image restoration, image segmentation, and texture modeling, in: C. Bouman, E. Miller, I. Pollak (Eds.), Computational Imaging, Vol. IV, SPIE, 2006. 
[17] J. Darbon, M. Sigelle, Image restoration with discrete constrained total variation, part I: fast and exact optimization, Journal of Mathematical Imaging and Vision Online first: 10.1007/s10851-006-8803-0.

[18] J. Garnett, T. Le, Y. Meyer, L. Vese, Image decompositions using bounded variation and homogeneous Besov spaces, Tech. Rep. CAM Report 05-57, UCLA (2005).

[19] D. Goldfarb, W. Yin, Second-order cone programming methods for total variation based image restoration, SIAM Journal on Scientific Computing 27 (2) (2005) 622-645.

[20] A. Haddad, Y. Meyer, Variational methods in image processing, Tech. Rep. CAM Report 04-52, UCLA (2004).

[21] S. Kindermann, S. Osher, Saddle point formulation for a cartoon-texture decomposition, Tech. Rep. CAM Report 05-42, UCLA (2005).

[22] S. Kindermann, S. Osher, J. Xu, Denoising by BV-duality, Journal of Scientific Computing $28(2-3)(2006)$ 411-444.

[23] T. Le, L. Lieu, L. Vese, $B V$ and dual of $B V$ image decomposition models and minimization algorithms, Tech. Rep. CAM Report 05-13, UCLA (2005).

[24] T. Le, L. Vese, Image decomposition using total variation and $\operatorname{div}(B M O)$, SIAM Journal on Multiscale Modeling and Simulation 4 (2) (2005) 390-423.

[25] L. Lieu, Contribution to problems in image restoration, decomposition, and segmentation by variational methods and partial differential equations, Ph.D. thesis, UCLA (2006).

[26] L. Lieu, L. Vese, Image restoration and decomposition via bounded total variation and negative Hilbert-Sobolev spaces, Tech. Rep. CAM Report 05-33, to appear in Applied Mathematics and Optimization, UCLA (2005).

[27] Y. Meyer, Oscillating patterns in image processing and nonlinear evolution equations, Vol. 22 of University Lecture Series, AMS, 2002.

[28] Mosek ApS Inc., The Mosek optimization tools, ver 4. (2006).

[29] M. Nikolova, Minimizers of cost-functions involving nonsmooth data-fidelity terms, SIAM Journal on Numerical Analysis 40 (3) (2002) 965-994.

[30] M. Nikolova, A variational approach to remove outliers and impulse noise, Journal of Mathematical Imaging and Vision 20 (1-2) (2004) 99-120.

[31] M. Nikolova, Weakly constrained minimization. application to the estimation of images and signals involving constant regions, Journal of Mathematical Imaging and Vision 21 (2) (2004) 155-175.

[32] S. Osher, A. Sole, L. Vese, Image decomposition and restoration using total variation minimization and the $H^{-1}$ norm, SIAM Journal on Multiscale Modeling and Simulation 1 (2003) 349-370. 
[33] L. Rudin, S. Osher, E. Fatemi, Nonlinear total variation based noise removal algorithms, Physica D 60 (1992) 259-268.

[34] J.-L. Starck, M. Elad, D. Donoho, Image decomposition via the combination of sparse representation and a variational approach, IEEE Transactions on Image Processing 14 (10) (2005) 1570-1582.

[35] L. Vese, S. Osher, Modeling textures with total variation minimization and oscillating patterns in image processing, Journal of Scientific Computing 19 (13) (2003) 553-572.

[36] W. Yin, T. Chen, X. S. Zhou, A. Chakraborty, Background correction for cDNA microarray image using the $T V+L^{1}$ model, Bioinformatics 21 (10) (2005) 24102416 .

[37] W. Yin, D. Goldfarb, S. Osher, Image cartoon-texture decomposition and feature selection using the total variation regularized $L^{1}$ functional, in: Variational, Geometric, and Level Set Methods in Computer Vision, Vol. 3752 of Leture Notes in Computer Science, Springer, 2005, pp. 73-84.

[38] W. Yin, D. Goldfarb, S. Osher, On the multiscale decomposition by the TV$L^{1}$ model, Tech. Rep. CORC Report TR2006-03, to appear in SIAM MMS, Columbia University (2006).

[39] W. P. Ziemer, Weakly Differentiable Functions: Sobolev Spaces and Functions of Bounded Variation, Graduate Texts in Mathematics, Springer, 1989. 